\title{
Pseudo-natural products and natural product-inspired methods in chemical biology and drug discovery Michael Grigalunas ${ }^{1}$, Annina Burhop ${ }^{1,2}$, Andreas Christoforow ${ }^{1,2}$ and Herbert Waldmann ${ }^{1,2}$
}

\begin{abstract}
Through evolution, nature has provided natural products (NPs) as a rich source of diverse bioactive material. Many drug discovery programs have used nature as an inspiration for the design of NP-like compound classes. These concepts are guided by the prevalidated biological relevance of NPs while going beyond the limitations of nature to produce chemical matter that could have unexpected or novel bioactivities. Herein, we discuss, compare, and highlight recent examples of NP-inspired methods with a focus on the pseudo-NP concept.

Addresses

${ }^{1}$ Department of Chemical Biology, Max-Planck-Institute of Molecular Physiology, Otto-Hahn-Straße 11, 44227 Dortmund, Germany

${ }^{2}$ Faculty of Chemistry and Chemical Biology, Technical University

Dortmund, Otto-Hahn-Straße 6, 44221 Dortmund, Germany
\end{abstract}

Corresponding author: Waldmann, Herbert (herbert.waldmann@mpidortmund.mpg.de)

Current Opinion in Chemical Biology 2020, 56:111-118

This review comes from a themed issue on Next Generation Therapeutics

Edited by Gonçalo Bernardes and Raphaël Rodriguez

For a complete overview see the Issue and the Editorial

Available online 1 May 2020

https://doi.org/10.1016/j.cbpa.2019.10.005

1367-5931/@ 2019 Published by Elsevier Ltd.

Keywords

Small molecules, Natural products, Chemical biology, Ring distortion, Biology-oriented synthesis, Pseudo-natural products.

\section{Introduction}

Approaches that inspire new molecular discovery programs are at the core of chemical biology and medicinal chemistry. As the mode of action of small molecules compared to most genetic approaches can be rapid, tunable, conditional, and reversible [1], the development of selective probes is of high value for biological applications. A key challenge that arises is the identification of areas in chemical space, which are relevant for bioactivity [2], because the possible number of small molecules is at least $10^{60}$, too many to ever be synthesized, nonetheless biologically evaluated [3].
Natural products (NPs) contain privileged molecular scaffolds with inherent biological relevance, having themselves evolved to fulfill specific biological functions within the context of signaling pathways and protein interactions [4]. These frameworks are, therefore, 'prevalidated' representations of nature's exploration of biologically relevant chemical space. While numerous NP-based drug discovery programs have successfully used NPs or their close derivatives as endpoints [5], other methods have thrived on using NPs as starting points to inspire new bioactive compound collections. In this review, recent developments in methods that are inspired by nature will be discussed with a focus on the recently disclosed pseudo-NPs concept.

\section{Ring distortion strategy - complexity-to- diversity (CtD)}

An elegant approach to exploit nature's pre-validated and rich pool of biologically relevant molecular scaffolds was introduced in 2013 as a ring distortion/modification strategy by Hergenrother and co-workers [6**]. Instead of regarding the complex structures of NPs as the final product in a synthesis or drug discovery effort, they are set as privileged starting points for the exploration of NPderived chemical space through appropriate chemical modifications of orthogonal functional characteristics embedded in the chosen NPs. In contrast to the traditional optimization efforts, which intend to improve potency or drug-like properties of a NP, the ring distortion/ modification approach aims for significant disruption of the overall topology of the parent NP by manipulation of core ring systems. This approach aims for a high degree of scaffold diversity and is, hence, referred to as the complexity-to-diversity $(\mathrm{CtD})$ strategy.

$\mathrm{CtD}$ is inspired by the biosynthetic pathways leading to complex NPs where common intermediates are transformed by diverse arrays of various enzymes to generate compounds that are distinct from each other. Transferring this logic into the hands of organic chemists, enzymes become chemoselective reagents, which enable the strategic manipulation of a suitable NP through ring-cleavage, ring-rearrangement, ring-fusion and modification of ring-size (expansion or contraction), and/or its oxidation state (e.g., aromatization), as illustrated in Figure 1a. 


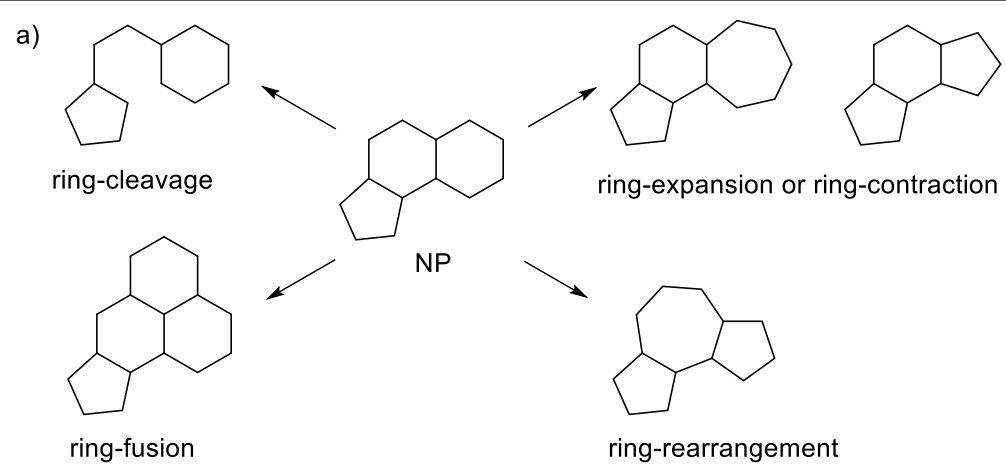

b)

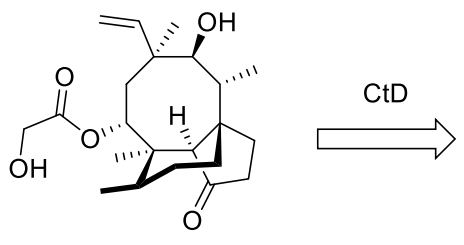

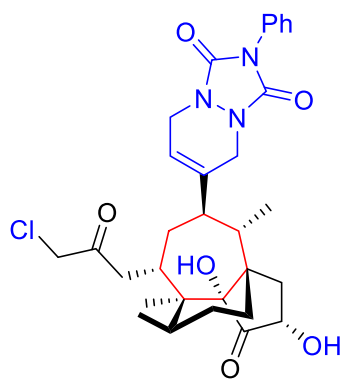

ferroptocide (thiredoxin inhibitor)

c)<smiles>[R]c1ccc2nccc([C@@H](O)C3CC4CCN3C4C=C)c2c1</smiles>

xa, $\mathrm{R}=\mathrm{H}$, cinchonine $\mathbf{x b}, \mathrm{R}=\mathrm{OMe}$, quinidine

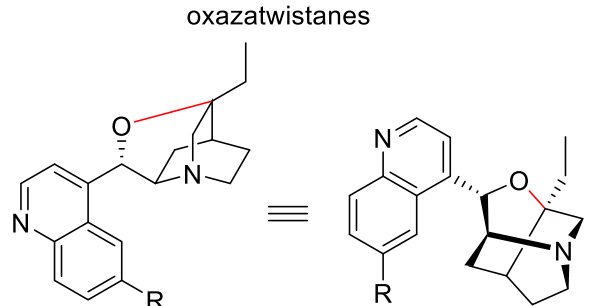<smiles>[R]c1cc([C@@H](CN2CC3CCC2(CC)C3)O[Na])c2c([R])c([R])c([R])cc2n1</smiles>

(autophagy inhibitor)

Current Opinion in Chemical Biology

(a) General depiction of the ring distortion strategy. (b) Ring contraction (highlighted in red) and diversification (highlighted in blue) of pleuromutilin to give a thiredoxin inhibitor, ferroptocide. (c) Ring-fusion of cinchona alkaloids to provide oxazatwistanes (highlighted in red). Further diversification (highlighted in blue) and phenotypic screening provided the autophagy inhibitor oxautin-1. 
A synthetic proof of concept was outlined by the diversification of several NPs, including gibberellic acid [6**], adrenosterone [6**], quinine [6**], abietic acid [7], yohimbine [8], sinomenine [9], and lycorine [10]. A recent example demonstrated the biological relevance of $\mathrm{CtD}$ by the diversification of pleuromutilin and subsequent biological evaluation (Figure 1b) [11*]. In total, 29 structurally diverse and highly complex derivatives of pleuromutilin were synthesized using $\mathrm{CtD}$ and screened in a phenotypic assay for anticancer activity. A ringcontraction product of pleuromutilin was further elaborated to ferroptocide and was found to rapidly induce ferroptotic death of cancer cells by inhibiting thioredoxin.

The biological relevance of the CtD approach was further verified by Laraia et al., focusing on the diversification of oxazatwistanes (Figure 1c) [12*]. The synthesis commenced with an intramolecular etherification of cinchonine or quinidine to install the ringfused non-natural oxazatricyclo[4.4.0.0]decane scaffold. Subsequent metal-catalyzed cross-coupling reactions and $\mathrm{C}-\mathrm{H}$ functionalization were used to further modify the quinoline core to afford a total of $47 \mathrm{com}$ pounds that were subjected for biological evaluation in a range of phenotypic assays. The identification of several autophagy inhibitors, an activity not exhibited by cinchona alkaloids at concentrations up to $30 \mu \mathrm{M}$ [13], demonstrated that significant changes in the topology of NPs might lead to novel NP-inspired molecular frameworks that are endowed with different bioactivity profiles than their natural predecessors.

\section{Biology-oriented synthesis}

Biology-oriented synthesis (BIOS) is a design principle to generate small molecules as tools for the study of complex biological systems [4,14,15**]. In nature, evolutionary conservation of molecular architectures leads only to a limited number of possible smallmolecule binding sites [16]. Analogously, NPs have come from the function-driven evolution of biosynthetic pathways. Whereas the surrounding substitution patterns can be highly diverse, the core scaffolds are conserved. BIOS uses NPs as prevalidated starting points in the development of bioactive molecules. From a chemoinformatic structural analysis of NPs [2], simplified core scaffolds have been identified that may transfer biologically relevant characteristics to the resulting synthetic molecule [17]. Diversification of substituent patterns can lead to specific selectivity profiles and can provide NP-inspired compound collections that are more elaborate and have enriched bioactivity relative to combinational libraries. Therefore, the size of the compound collection can be reduced while increasing bioactivity hit rates.

The application of BIOS in providing compounds for the development of optimized structures, for example, in potency or pharmacodynamic properties, has been shown in various examples. Secoyohimane NPs such as rychnophylline (Figure 2a) are known for their neurite growth-promoting properties [18-20]. The simplification to the spiro-core scaffold composed of four connected rings allowed the synthesis of five derivatives, which are active in neurite growth assays [21]. Similarly, derivatives of the NP sominone (Figure 2b) with the dehydro- $\delta$-lactone motif were identified as potent inhibitors of the Hedgehog signaling pathway [22]. Overall, the BIOS approach has structurally and, thereby, synthetically simplified NPs to provide small molecules that have new or improved bioactivities.

\section{Pseudo-natural products}

While several approaches have been developed to produce novel bioactive compound collections, they may have limitations. The $\mathrm{CtD}$ approach gives rapid access to diverse biological space through chemical derivatization of NPs. However, the use of NPs as a starting point may present a chemical limitation because the availability of sufficient quantities of many NPs is restricted. Nevertheless, complex polycyclic scaffolds that are NPlike can be directly synthesized and distorted [23]. In BIOS, NPs inspire the synthesis of structurally simplified but biologically relevant compound collections. NPs occupy a large yet limited amount of chemical space [24]. However, NP-like chemical space is significantly larger than existing NP scaffolds and therefore presents a chemical limitation of BIOS. From a biological viewpoint, these compounds may retain similar bioactivities to the structurally related parent NP, thereby limiting the exploration of biological space [17].

Methods that allow for rapid access to biologically pertinent chemical space while retaining biological relevance to NPs may provide useful compound collections. This matching led to the development of the pseudo-NP approach [25**,26**] in which bioactive compound classes are obtained through combining strategies that take inspiration from nature, such as BIOS, with principles that efficiently cover a vast amount of chemical space, such as fragment-based compound design. In a cheminformatics analysis, more than $180,000 \mathrm{NPs}$ were analyzed and simplified to approximately 2,000 NP-based fragment classes in which the properties of the NPs were retained [27]. It was hypothesized that the de novo combination and arrangement of the NP fragments could give unprecedented combinations or fusion patterns that may provide novel scaffolds that are significantly different from NPs and other NP-based collections (Figure 3a). These compounds could retain biological and chemical relevance to NPs but extend beyond the biologically relevant chemical space explored by nature. The new compound classes resemble NPs but are not accessible through existing biosynthetic pathways. Therefore, they 


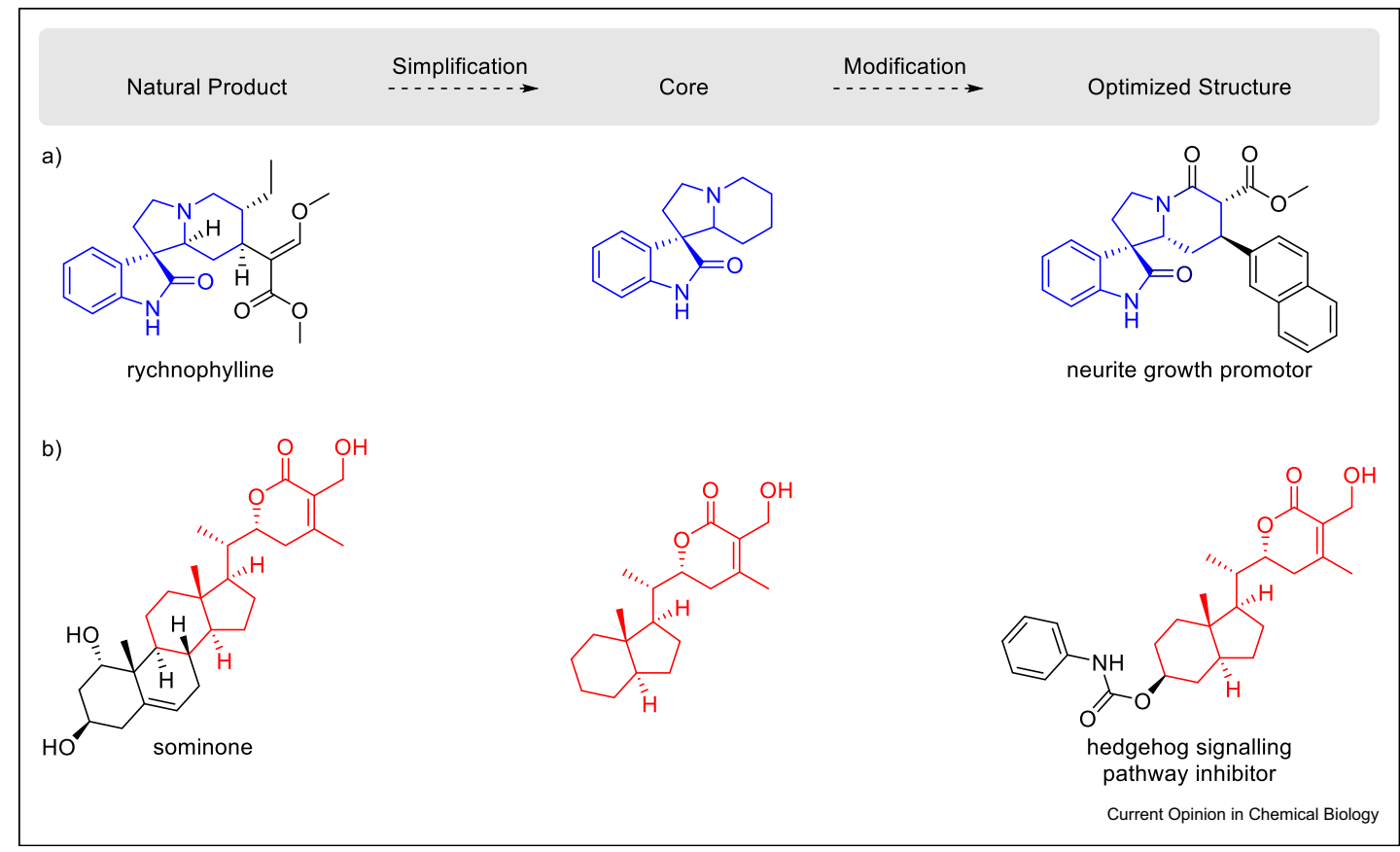

The BIOS approach with (a) rychnophylline and (b) sominone, which provided compounds that promote neurite growth and inhibit the Hedgehog signaling pathway, respectively.

were termed 'pseudo-natural products' (pseudo-NPs). The term pseudo-NPs has been previously used by Suga et al. and Oshima et al. to describe cyclic peptides [28,29] and products from intercepted biosynthetic pathways $[30,31]$, respectively.

The general design criteria of pseudo-NP compound classes should combine NP-fragments with diverse bioactivities, providing structures that have chirality and complementary heteroatom content, such as oxygen and nitrogen. Furthermore, the merging of biosynthetically unrelated fragments may be advantageous for inducing new bioactivities. Further principles can provide expansive libraries based on different connectivity patterns of the same NP-fragment combinations. For example, the chromane and tropane fragment could be combined in different ways to provide spiro-fused or edge-fused compounds (Figure $3 \mathrm{bi}$ ). Other compounds could retain the same connectivity pattern but have different connection points (Figure 3 bii). Finally, more than two fragments could be connected in various patterns (Figure 3biii).

The pseudo-NP design principle may lead to new scaffolds that could have unexpected or unprecedented biological targets. To biologically evaluate these compounds, unbiased assays can be used to monitor entire biological systems, such as signaling cascades and phenotypic changes, instead of individual targets [32].
Beyond individual phenotypes, multiparametric highcontent image screening technologies, such as the 'cell painting' assay, can be used to assist hypothesis generation of the biological target or pathway that is being affected [33]. In addition, such phenotypic profiling enables evaluation of library performance diversity and activity distributions across sub-library classes, hence allowing informed library designs toward high performance diversity or hit expansion into promising structure classes [34].

The first example of the pseudo-NP concept provided an unprecedented combination of a chromane and a tetrahydropyrimidone (THPM) fragment through a bridged fusion connection (Figure 4a) [25**]. The 'chromopynone' library was synthesized in a telescoped manner via a variant of the Biginelli reaction followed by a single-flask, multistep reaction cascade. Biological evaluation of the chromopynone collection showed inhibition in glucose uptake assays. $(-)-(R, R)$-Chromopynone- 1 was the most potent compound in the library, selectively inhibited glucose transporters GLUT-1 and GLUT-3, and inhibited cancer cell growth. It is interesting to note that the bioactivity of the pseudo-NP was neither shared by 2,801 compounds that contain the chromane fragment or substructure nor 1,270 compounds containing the THPM fragment or substructure in glucose uptake assays. Therefore, the combination of chromane and THPM fragments provided novel 


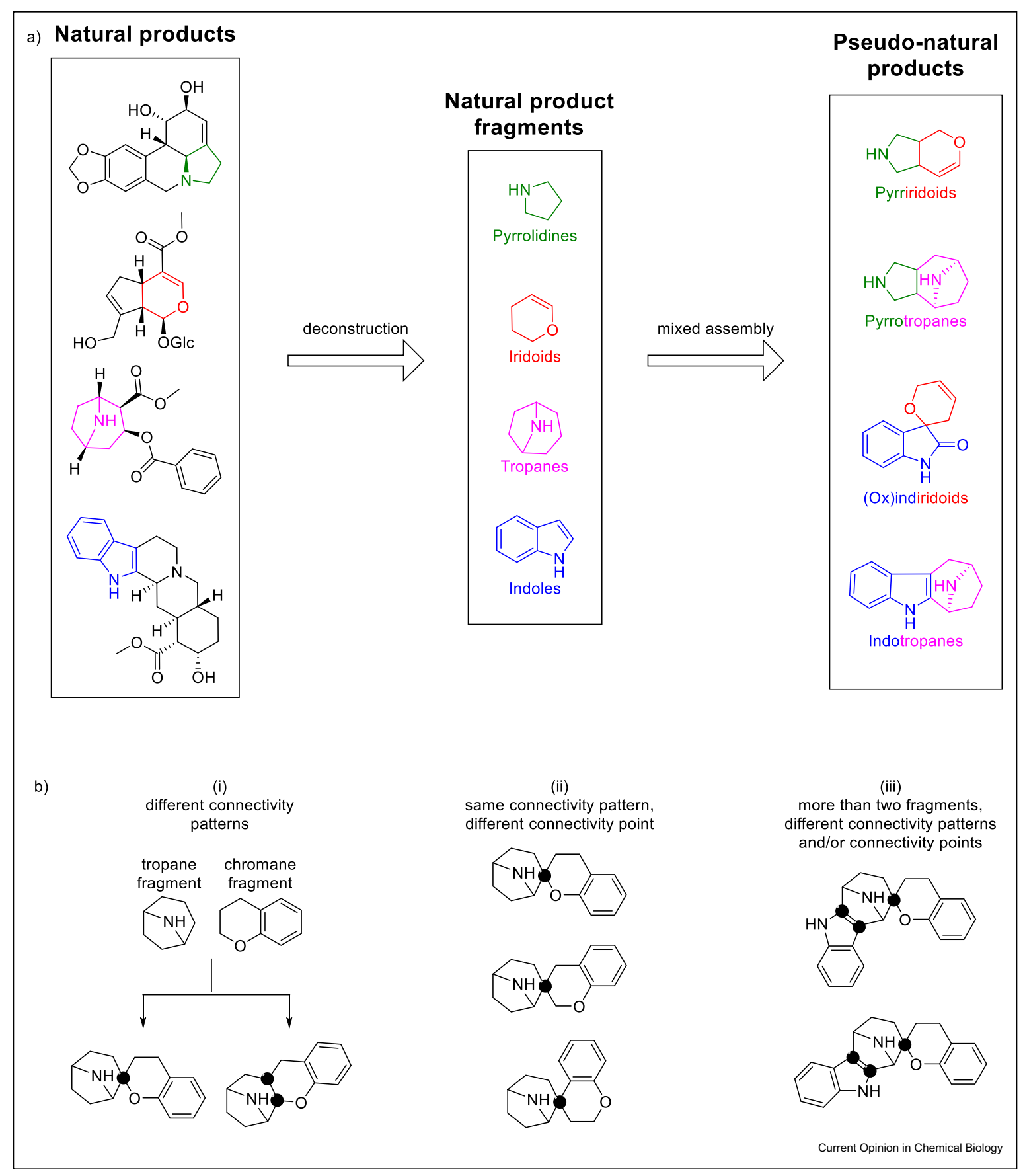

(a) Design of pseudo-NPs through deconstruction of NPs and mixed reassembly. (b) Variation of (i) connectivity patterns, (ii) connection points, and (iii) the combination of more than two NP-fragments in pseudo-NP design.

bioactivity that is unrelated to the two independent NP fragments.

Indotropanes were reported as a class of pseudo-NPs that combine the biosynthetically unrelated fragments indole and tropane through a bipodal connection (Figure 4b). Cu-catalyzed $3+2$ cycloaddition reactions were used to provide a stereogenically complex library of indotropanes [35]. After biological evaluation, myokinasib was found to induce extensive cell rounding and to impair cell division, causing multinucleation [36*]. It was hypothesized that this phenotype may be due to failed cytokinesis. From a kinase panel and several validation experiments, the phenotype could be attributed 


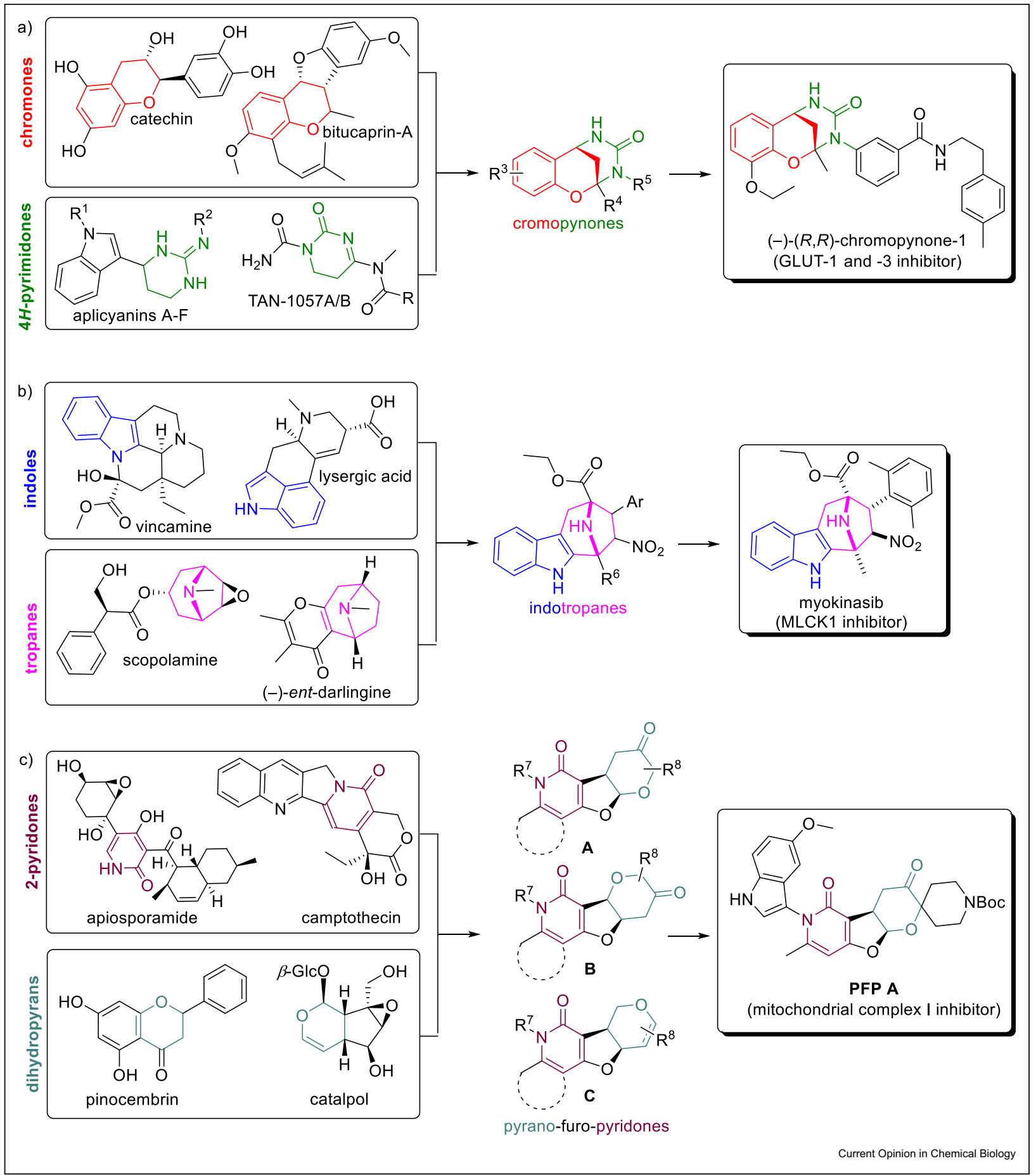

Design and implementation of the pseudo-NP method to provide (a) chromopynone, (b) indotropane, and (c) pyrano-furo-pyridone compound classes. Unique bioactivities were found within the compound classes of GLUT-1/-3, MLCK1, and mitochondrial complex I inhibition, respectively.

to the inhibition of MLCK1. Myokinasib is the first selective, isoform-specific inhibitor of MLCK1. Relative to typical ATP-competitive kinase inhibitors, which are usually flat and aromatic, myokinasib is stereogenically complex and has a three-dimensional shape. The unprecedented combination of the indole and tropane led to a pseudo-NP that has unprecedented bioactivity and is a novel chemotype for kinase inhibition. 
2-Pyridone and dihydropyran NP fragments comprise several unique structures and biological activities. Using either a Pd-catalyzed Tsuji-Trost cascade, a Pd-catalyzed Tsuji-Trost oxa-Michael cascade or a quinine-mediated Michael transacetalization cascade, these rarely related NP fragments could be fused in three distinct bipodal attachment point isomers to provide a compound library of pyrano-furo-pyridones (PFPs) (Figure 4c) [37*]. Morphological profiling by means of a cell painting assay enabled the prioritization of substructure class $\mathbf{A}$ and guided the discovery and identification of PFP $\mathbf{A}$ as a structurally novel inhibitor of mitochondrial complex I. Further analysis of the cell painting data indicated general trends in structure-phenotype relation, which could be subsequently confirmed in a structure-activity relationship study by a cell-based fluorogenic assay measuring the production of mitochondrial superoxide (MitoSOX Red assay).

\section{Conclusions and outlook}

Nature continues to be a source of inspiration for the design of novel bioactive compound collections. The $\mathrm{CtD}$ approach synthetically mimics enzymatic processes by chemically functionalizing and distorting NPs to structurally diverse compound collections. In BIOS, NPs inspire the synthesis of structurally simplified compound collections that are enriched in bioactivity. Nevertheless, the CtD and BIOS approaches may be limited due to their focus on specific NP scaffolds as starting points.

The pseudo-NPs principle aims to combine the biological relevance of NP-guided methods with the rapid chemical exploration of fragment-based ligand discovery through the deconstruction of NPs to fragments and their mixed reassembly to provide scaffolds that are unprecedented in nature. The three classes of pseudoNPs discussed here provide support that pseudo-NPs may display novel and unexpected bioactivities that differ from the parent NPs. We expect that the design principle of pseudo-NPs will provide compound collections that will have significant value toward the exploration of biologically relevant chemical space, eventually disclosing novel molecular architectures eliciting potentially unprecedented modes of actions, which can be developed further to chemical probes or serve as privileged starting points in chemical biology and drug discovery projects.

\section{Conflict of interest statement}

Nothing declared.

\section{Acknowledgements}

This work was supported by the Max-Planck-Gesellschaft. M. Grigalunas is grateful to the Alexander von Humboldt Foundation for a postdoctoral fellowship.

\section{References}

Papers of particular interest, published within the period of review, have been highlighted as:

* of special interest

** of outstanding interest

1. Zamir E, Bastiaens PIH: Reverse engineering intracellular biochemical networks. Nat Chem Biol 2008, 4:643-647.

2. Koch MA, Schuffenhauer A, Scheck M, Wetzel S, Casaulta M, Odermatt A, Ertl P, Waldmann $\mathrm{H}$ : Charting biologically relevant chemical space: a structural classification of natural products (SCONP). Proc Natl Acad Sci 2005, 102:17272-17277.

3. Bohacek RS, McMartin C, Guida WC: The art and practice of structure-based drug design: a molecular modeling perspective. Med Res Rev 1996, 16:3-50.

4. Wetzel S, Bon RS, Kumar K, Waldmann H: Biology-oriented synthesis. Angew Chem Int Ed 2011, 50:10800-10826.

5. Li JW, Vederas JC: Drug discovery and natural products: end of an era or an endless frontier? Science 2014, 325:161-166.

6. Huigens III RW, Morrison KC, Hicklin RW, Flood Jr TA,

** Richter MF, Hergenrother PJ: A ring-distortion strategy to construct stereochemically complex and structurally diverse compounds from natural products. Nat Chem 2013, 5 : 195-202.

This article is the seminal report of the ring distortion strategy in which the concept is outlined. Three readily available NPs were distorted to provide compounds that retain the properties of NPs while being structurally diverse relative to the parent NP and other compounds within the collection to provide a chemical proof of concept.

7. Rafferty RJ, Hicklin RW, Maloof KA, Hergenrother PJ: Synthesis of complex and diverse compounds through ring distortion of abietic acid. Angew Chem Int Ed 2014, 53:220-224.

8. Paciaroni NG, Ratnayake R, Matthews JH, Norwood VM Arnold AC, Dang LH, Luesch H: A tryptoline ring-distortion strategy leads to complex and diverse biologically active molecules from the indole alkaloid yohimbine. Chem Eur 2017, 23:4327-4335.

9. Garcia A, Drown BS, Hergenrother PJ: Access to a structurally complex compound collection via ring distortion of the alkaloid sinomenine. Org Lett 2016, 18:4852-4855.

10. Tasker SZ, Cowfer AE, Hergenrother PJ: Preparation of structurally diverse compounds from the natural product lycorine. Org Lett 2018, 20:5894-5898.

11. Llabani E, Hicklin RW, Lee HY, Motika S E, Crawford LA, Weerapana E, Hergenrother PJ: Diverse compounds from pleuromutilin lead to a thioredoxin inhibitor and inducer of ferroptosis. Nat Chem 2019, 11:521-532.

Applying the ring distortion strategy to the NP pleuromutilin provided ferroptocide, which induces ferroptotic death of cancer cells by inhibiting thioredoxin. This article gives support for the biological relevance of the products generated from the ring distortion method.

12. Laraia L, Onsawa K, Konstantinidis G, Robke L, Wu Y, Kumar K Waldmann $\mathrm{H}$ : Discovery of novel cinchona-alkaloid-inspired oxazatwistane autophagy inhibitors. Angew Chem Int Ed 2017, 56:2145-2150.

Following the ring distortion strategy, ring fusion and diversification of quinidine resulted in a nanomolar inhibitor of autophagy. This further verifies the biological potential of products obtained by the ring distortion method.

13. Golden EB, Cho H, Hofman FM, Louie SG, Schönthal AH, Chen TC: Quinoline-based antimalarial drugs: a novel class of autophagy inhibitors. Neurosurg Focus 2015, 38:E12.

14. Van Hattum $\mathrm{H}$, Waldmann $\mathrm{H}$ : Biology-oriented synthesis: harnessing the power of evolution. J Am Chem Soc 2014, 136 11853-11859.

15. Karageorgis G, Waldmann H: Guided by evolution: biology** oriented synthesis of bioactive compound classes. Synthesis 2019, 51:55-66. 
This review outlines the principles of biology-oriented synthesis and provides examples and an outlook for the design of NP-inspired compound collections.

16. Dobson CM: Chemical space and biology. Nature 2004, 432 824-828.

17. Crane EA, Gademann K: Capturing biological activity in natural product fragments by chemical synthesis. Angew Chem Int Ed 2016, 55:3882-3902.

18. Praveen V, Reddy RG, Duy D, Chakravarty S, Chandrasekhar S, Grée R: Bioorganic \& medicinal chemistry letters synthesis and neurite growth evaluation of new analogues of honokiol, a neolignan with potent neurotrophic activity. Bioorg Med Chem Lett 2012, 22:1439-1444.

19. Cheng X, Harzdorf N, Khaing Z, Kang D, Camelio AM, Shaw T, Schmidt CE, Siegel D: Neuronal growth promoting sesquiterpene - neolignans; syntheses and biological studies. Org Biomol Chem 2012, 10:383-393.

20. Rawat M, Gama Cl, Matson JB, Hsieh-Wilson LC: Neuroactive chondroitin sulfate glycomimetics. J Am Chem Soc 2008, 130 : 2959-2961.

21. Antonchick AP, Lopez-Tosco S, Parga J, Sievers S, Schürmann M, Preut H, Höing S, Schöler HR, Sterneckert J, Rauh D, Waldmann $\mathrm{H}$ : Enantioselective catalytic synthesis of neurite growth-promoting secoyohimbanes. Chem Biol 2013, 20:500-509.

22. Sheremet M, Kremer L, Bauer JO: Biology-oriented synthesis of a withanolide-inspired compound collection reveals novel modulators of Hedgehog signaling. Angew Chem Int Ed 2015, 54:5596-5602.

23. Foley DJ, Craven PGE, Collins PM, Doveston RG, Aimon A, Talon R, Churcher I, von Delft F, Marsden SP, Nelson A: Synthesis and demonstration of the biological relevance of $\mathrm{sp}^{3}$ rich scaffolds distantly related to natural product frameworks. Chem Eur J 2017, 23:15227-15232.

24. Pye CR, Bertin MJ, Lokey RS, Gerwick WH, Linington RG: Retrospective analysis of natural products provides insights for future discovery trends. Proc Natl Acad Sci 2017, 114: $5601-5606$.

25. Karageorgis G, Reckzeh ES, Ceballos J, Schwalfenberg M * Sievers S, Ostermann C, Pahl A, Ziegler S, Waldmann H: Chromopynones are pseudo natural product glucose uptake inhibitors targeting glucose transporters GLUT-1 and -3. Nat Chem 2018, 10:1103-1111.

This is the seminal publication of the pseudo-NP concept in which chromone and tetrahydropyrimidone fragments are combined $(-)-(R, R)$-Chromopynone-1 was found to inhibit the growth of cancer cells by selectively inhibiting glucose transporters GLUT-1 and -3 and provides a proof of principle for the pseudo-NP method.

26. Karageorgis G, Foley DJ, Laraia L, Waldmann H: Pseudo-natural products: principle and design. Nat Chem 2019 (submitted). In this concept piece, the rationale and principle of the pseudo-NPs method is outlined for the discovery of bioactive small molecules. Proof-of-concept examples are provided as well as design principles for future library design.

27. Over B, Wetzel S, Grütter C, Nakai Y, Renner S, Rauh D, Waldmann H: Natural-product-derived fragments for fragment-based ligand discovery. Nat Chem 2013, 5:21-28.
28. Goto Y, Ito Y, Kato Y, Tsunoda S, Suga H: One-pot synthesis of azoline-containing peptides in a cell-free translation system integrated with a posttranslational cyclodehydratase. Chem Biol 2014, 21:766-774.

29. Ozaki T, Yamashita K, Goto Y, Shimomura M, Hayashi S, Asamizu S: Dissection of goadsporin biosynthesis by in vitro reconstitution leading to designer analogues expresed in vivo. Nat Commun 2017, 8:1-13.

30. Asai T, Tsukada K, Ise S, Shirata N, Hashimoto M, Fujii I, Gomi K, Nakagawara K, Kodama EN, Oshima Y: Use of a biosynthetic intermediate to explore the chemical diversity of pseudo-natural fungal polyketides. Nat Chem 2015, 7 $737-743$

31. Kikuchi H, Ichinohe K, Kida S, Murase S, Yamada O, Oshima Y: Monoterpene indole alkaloid-like compounds based on diversity-enhanced extracts of iridoid-containing plants and their immune checkpoint inhibitory activity. Org Lett 2016, 18 5948-5951.

32. Mullard A: The phenotypic screening pendulum swings. Nat Rev 2015, 14:807-809.

33. Caicedo JC, Cooper S, Heigwer F, Warchal S, Qiu P, Molnar C, Vasilevich AS, Barry JD, Bansal HS, Rohban M, Hung J, Hennig H, Concannon J, Smith I, Clemons PA, Singh S, Rees P Horvath $P$, Linington RG, Carpenter AE: Data-analysis strategies for image-based cell profiling. Nat Methods 2017, 14: 849-863.

34. Wawer MJ, Li K, Gustafsdottir SM, Ljosa V, Bodycombe NE, Marton MA, Hon CS, Duvall JR, Wilson JA, Bittker JA, Dančík V, Narayan R, Subramanian A, Winckler W, Golub TR Carpenter AE, Shamji AF, Schreiber SL, Clemons PA: Toward performance-diverse small-molecule libraries for cellbased phenotypic screening using multiplexed highdimensional profiling. Proc Natl Acad Sci 2014, 111: $10911-10916$.

35. Narayan R, Bauer JO, Strohmann C, Antonchick AP, Waldmann H: Catalytic enantioselective synthesis of functionalized tropanes reveals novel inhibitors of Hedgehog signaling. Angew Chem Int Ed 2013, 52:12892-12896.

36. Schneidewind T, Kapoor S, Garivet G, Karageorgis G, Narayan R Vendrell-Navarro G, Antonchick AP, Ziegler S, Waldmann H: The pseudo natural product myokinasib is a myosin light chain kinase 1 inhibitor with unprecedented chemotype. Cell Chemical Biol 2018, 26:512-523.

Identification of the pseudo-NP myokinasib as a selective kinase inhibitor is reported and further underscores the biological relevance of pseudo-NPs. This article provides evidence that the unique chemotypes of pseudo-NPs can have unexpected bioactivities.

37. Christoforow A, Wilke J, Binici A, Pahl A, Ostermann C, Sievers S, Waldmann $\mathrm{H}$ : Design, synthesis and phenotypic profiling of pyrano-furo-pyridone pseudo natural products. Angew Chem Int Ed 2019, https://doi.org/10.1002/anie.201907853.

A class of pyrano-furo-pyridone pseudo-NPs were synthesized and found to contain structurally novel inhibitors of mitochondrial complex I. A "cell painting" assay was used to guide the identification of biologically relevant compounds and highlights the potential of this assay to identify bioactive compounds that have novel chemotypes and/or are not linked to a specific target. 